\title{
Immunoglobulin and complement inclusions in peripheral blood polymorphonuclear leucocytes of patients with bronchial carcinoma
}

\author{
H. M. JANSEN ${ }^{2}$, T. H. THE ${ }^{1}$, G. C. DE GAST ${ }^{1}$, H. A. HUIGES ${ }^{1}$, M. T. ESSELINK ${ }^{1}$, \\ A. M. VAN DER WAL ${ }^{2}$, AND N. G. M. ORIE ${ }^{2}$ \\ From the Clinical Immunology Unit ${ }^{1}$, and the Pulmonary Division ${ }^{2}$, Department of Medicine, \\ University of Groningen, The Netherlands
}

Jansen, H. M., The, T. H., de Gast, G. C., Huiges, H. A., Esselink, M. T., Van der Wal, A. M., and Orie, N. G. M. Thorax, 32, 706-710. Immunoglobulin and complement inclusions in peripheral blood polymorphonuclear leucocytes of patients with bronchial carcinoma. Inclusions in peripheral blood polymorphonuclear leucocytes (PMN) of 23 patients with bronchial carcinoma, of 11 individuals with chronic obstructive lung disease (COLD), and of 11 healthy controls were studied by the indirect immunofluorescence technique for IgG and complement. The relationships of these inclusions to the clinical stage and histology of the malignant disease as well as the influence of subsequent therapy were studied.

Patients with disseminated bronchial carcinoma, stage III, had higher PMN scores than patients with localised disease, stage I. Both patient groups had higher scores than the two control groups. The differences were all statistically significant. There was no difference between the COLD group and the healthy controls.

PMN inclusion scores from the patient group with small- or large-cell undifferentiated carcinoma were higher than from patients with moderately differentiated or well differentiated squamous-cell carcinoma. The first two groups differed significantly from the controls; the latter group did not. Curative surgical therapy gave rise to a significant drop in PMN scores to normal values.

In four patients with regional lymph node metastasis (stage II) the PMN inclusions were related to evidence of recurrence at six months follow-up after operation and BCG immunostimulation.

It is concluded that the PMN inclusions are related to the extent of the disease, to the histological type, and to curative surgical therapy. The possible relation to immune complexes of tumour antigen-antibody origin is discussed.

Current knowledge points strongly to the presence of circulating immune complexes in many malignant as well as non-malignant diseases (Dixon, 1972; Oldstone et al., 1975). In systemic lupus erythematosus the possible significance of circulating complex phagocytosis by polymorphonuclear leucocytes (PMN) was recently studied by Steffelaar et al. (1976) using immunofluorescence techniques.

Immunofluorescence studies in animal tumour models have suggested that renal immune complex deposits indicate soluble circulating complexes of tumour-antigens and anti-tumour antibodies (Poskitt et al., 1974). In some patients with bronchial carcinoma or other solid tumours, there is also evidence for the presence of circulating immune complexes causing renal tissue injury as a complication of the patient's immune response to the tumour (Costanza et al., 1973; Lewis et al., 1971; Loughridge and Lewis, 1971). With the Raji cell radioimmune assay, Theofilopoulos et al. (1976) have demonstrated circulating immune complexes. 
In an attempt to elucidate the possible role of circulating immune complexes in patients with bronchial carcinoma, we studied the relation of immunoglobulin phagocytosis by PMN to the presence and progress of the disease.

\section{Patients and methods}

Twenty-three patients with histologically confirmed primary bronchial carcinoma were studied. The patients were classified in TNM stages, according to the criteria for lung carcinomas proposed by the International Union against Cancer (International Union against Cancer, 1974).

Histological tumour types included squamouscell carcinoma, adenocarcinoma, and small- and large-cell undifferentiated carcinoma.

The normal control group consisted of 11 individuals without a personal history of allergic disorder, recent bacterial or viral infections, or clinical evidence of malignant disease. In addition, 11 patients with chronic obstructive lung disease (COLD) and without a personal history or clinical evidence of malignant disease were included; five of them did have clinically proven bacterial bronchitis.

Blood samples for isolation of PMN were taken from the patients at the time that the diagnosis was established and, in the patients who underwent surgical therapy, also three weeks postoperatively. Polymorphonuclear leucocytes were isolated as the buffy coat from $10 \mathrm{ml}$ fresh venous blood which was immediately defibrinated in sterile flashes with $2 \mathrm{~mm}$ glass beads and subsequently spun down in a haematocrit tube. Red blood cells were lysed in a buffered $\mathrm{NH}_{4} \mathrm{Cl}$ solution at $0^{\circ} \mathrm{C}$ for $15 \mathrm{~min}$ and washed out in a Hepes buffered RPMI medium.

\section{IMMUNOFLUORESCENCE PROCEDURES}

Air-dried, cytospin preparations of the isolated PMN were made and fixed in $100 \%$ acetone. With this small modification the indirect immunofluorescence technique, described by Steffelaar et al. (1976), was used for demonstration of total IgG and total complement. All preparations had been frozen at $-70^{\circ} \mathrm{C}$ and thawed once. Slides were read in a Leitz-Orthoplan fluorescence microscope under incidental illumination using a xenon lamp for excitation. Positive inclusion cells were scored from $1000 \mathrm{PMN}$ in randomly chosen fields at $10 \times 40$ magnification. Scoring was done twice, by two observers, who were not aware of the diagnosis. Cells were recorded as positive only when at least five distinct fluorescent cytoplasma granules were seen (Fig. 1).

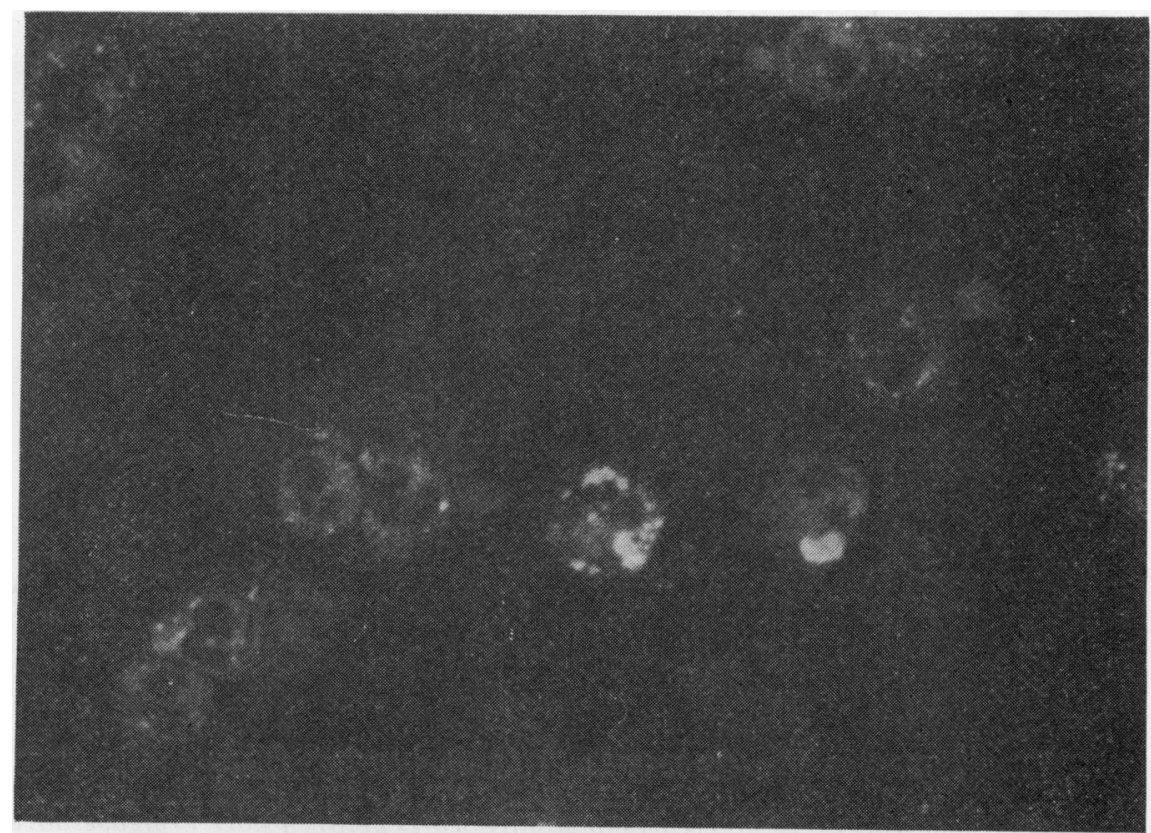

Fig. 1 Granular cytoplasmic inclusions in a PMN from a patient with squamous-cell bronchial carcinoma as seen in the immunofluorescence procedure with anti-IgG (X650). 
STATISTICAL ANALYSIS

Statistical analysis of the findings obtained was estimated by Wilcoxon's rank sum test. 2P values less than 0.05 were considered significant.

\section{Results}

Peripheral blood samples from 45 individuals have been examined. In Fig. 2 the data are presented. Scores of IgG inclusion positive PMN are shown in relation to different stages of bronchial carcinoma (median stage I, 35; median stage III, 79), to normal controls (median, 10), and to COLD controls (median, 12).

No significant difference in scores was observed between the latter two groups. No difference in scores was observed either in the COLD groups between individuals with and without bacterial bronchitis. In patients with bronchial carcinoma the PMN scores were higher and showed a relation to the extent of the disease. The differences between stage I, stage III, and both the control groups were significant $(2 \mathrm{P}<0.02$ and $2 \mathrm{P}<0.01)$. From six stage I patients PMN scores have been $\nRightarrow$ recorded three weeks after curative surgical $\vec{\circ}$ therapy. The scores were significantly lower (median, 9) $(2 \mathrm{P}<0.05)$ and did not differ from the control group.

There were very high positive PMN scores in stage III (patients with lymphatic and blood-borne disseminated disease).

From four individuals in stage II separate data are presented in the last two columns of Figure 2. In these cases surgical therapy appeared to be not curative after pathological investigation. PMN scores are given both pre- and post-operatively. In two patients PMN scores were much higher after surgical intervention. There was clinical recur-

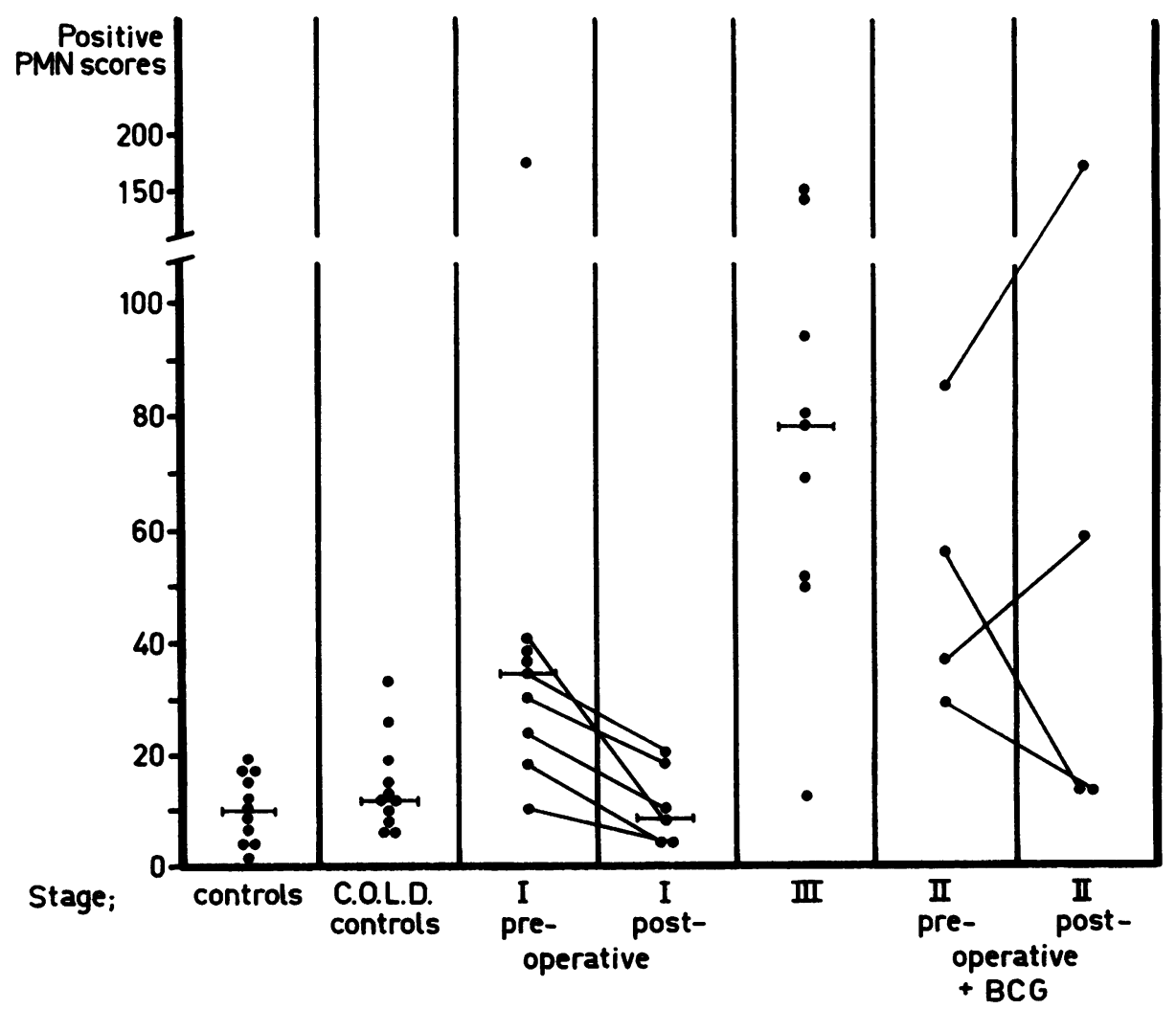

Fig. 2 Relation between positive IgG inclusion PMN scores and the different stages of bronchial carcinoma compared with the control groups and the influence of therapy. Cross bars indicate median scores. The differences in positive scores between stage I (preoperative), stage III, and both the control groups were significant $(2 \mathrm{P}<0.01$ and $2 \mathrm{P}<0.02)$. After surgery the scores in stage I patients were significantly lower $(2 \mathrm{P}<0 \cdot 05)$. There was no difference between the two control groups. 
rence of the disease in these two patients within six months in spite of BCG immunostimulation, started after the operation. The other two patients in this group, with lower PMN scores after surgery, did not show clinical recurrence after six months' follow-up.

Figure 3 shows the relationship between positive IgG inclusion PMN and the different histological patterns of bronchial carcinoma compared with the normal control group. There was a significant difference between PMN scores in undifferentiated carcinomas, in moderately differentiated squamouscell carcinomas, and in the control group $(2 \mathrm{P}<0.01)$. There was no significant difference between scores in well-differentiated squamous-cell carcinomas and in controls. There were only four patients with adenocarcinoma in this study. They seemed to have low PMN inclusion scores.
Results in the study of complement inclusion PMN were comparable to those from IgG, although there was more variability in the scores.

\section{Discussion}

Patients with bronchial carcinoma appeared to have higher scores of IgG and complement inclusions in their PMN than patients with chronic obstructive lung disease and normal controls. Furthermore, there was a positive correlation between positive PMN scores and the extent of the malignant disease, as well as in serial investigations after surgical therapy. PMN scores were also found to vary according to the histological patterns of bronchial carcinoma. No influence on PMN scores by bacterial respiratory infections were recorded in the COLD control group.

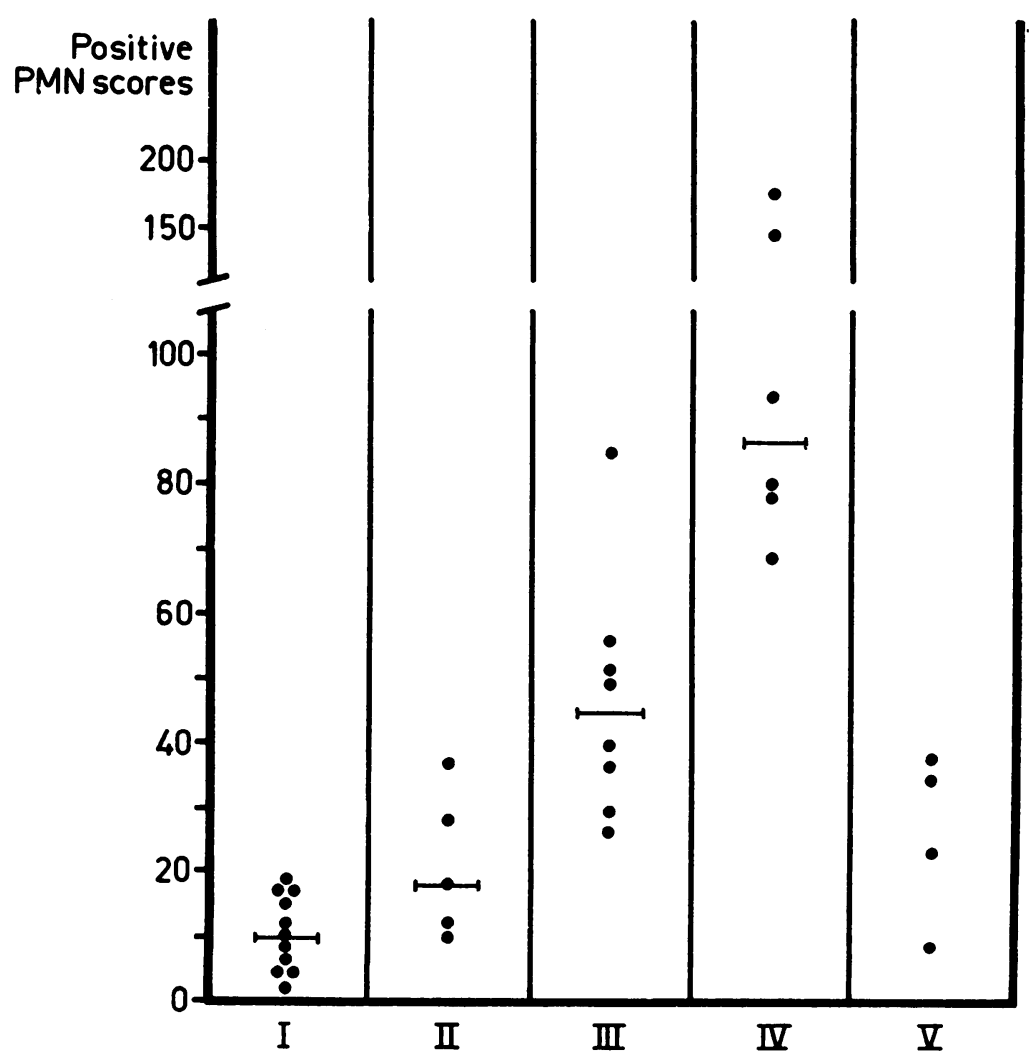

Fig. 3 Relation between positive IgG inclusion PMN scores and the different histological patterns of bronchial carcinoma compared with controls: I, controls; II, well-differentiated squamous-cell carcinoma; III, moderately differentiated squamous-cell carcinoma; IV, small-or large-cell undifferentiated carcinoma; $V$, adenocarcinoma. Cross bars indicate median scores. The differences between $I$ and $I I I, I$ and $I V$, and III and IV are significant $(2 \mathbf{P}<0.01)$. 
The studies of Sjögren et al. (1971) in mice tumour models have suggested that serum tumour antigen-antibody complexes are mediating blocking activity on effector functions of the cellular immune system. In tumour growth in man there is evidence of the same sort of interaction (Sjögren et al., 1972). Recent observations have shown that removing circulating serum factors by repeated plasmapheresis in malignant disease might tide the patients over a 'critical immunologic period' in the tumour-host relationship (Lancet, 1976). Theofilopoulos et al. (1976) have demonstrated circulating immune complexes in human sera in a variety of malignant diseases.

Our data are consistent with these observations and may indicate that PMN leucocytes of bronchial carcinoma patients may contain immune complexes, consisting of IgG antibodies against bronchial carcinoma-associated antigens.

It is not possible, using PMN phagocytosis for detection of circulating complexes, to exclude phagocytosis of immunoglobulin aggregates. The fact, however, that normal controls showed very low inclusion scores and the existence of normal immunoglobulin serum levels in all our patients' sera are in favour of immune complex phagocytosis. Furthermore, it has been reported by Onyewotu et al. (1974) and by Stühlinger et al. (1976) that soluble immune complexes inhibit the phagocytosis of aggregated IgG.

The specificity of the immune complexes with regard to bronchial carcinoma antigens remains to be proven, but the absence of an increased PMN score in the COLD patients with bacterial bronchitis renders the contribution of complexes caused by these infections unlikely.

It is obvious from the above discussion that PMN leucocytes may only phagocytise accumulated circulating complexes, and this may represent only a secondary phenomenon in bronchial carcinoma (Steffelaar et al., 1976). In the presence, however, of detectable PMN inclusions, related to tumour load, histology, and prognosis of the disease, one may use these criteria as an additional monitoring device in clinical and immunological observations of patients with bronchial carcinoma.

This study was supported by a grant from the 'Stichting Koningin Wilhelmina Fonds, Nederlandse Vereniging voor de Kankerbestrijding'.

\section{References}

Costanza, M. E., Pinn, V., Schwartz, R. S., and Nathanson, L. (1973). Carcinoembryonic antigen- antibody complexes in a patient with colonic carcinoma and nephrotic syndrome. New England Jour- $\frac{\bar{\omega}}{\bar{\omega}}$ nal of Medicine, 289, 520-522.

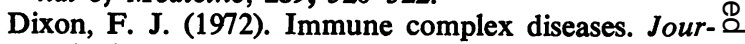
nal of Investigative Dermatology, 59, 413-415. के

International Union Against Cancer (1974). TNM-

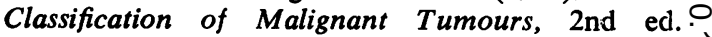
Geneva.

Lancet (1976). Plasmapheresis and immunosuppression $\stackrel{\circ}{\circ}$ (Editorial). Lancet, 1, 1113-1114.

Lewis, M. G., Loughridge, L. W., and Phillips, T. M. $\stackrel{\times}{\times}$ (1971). Immunological studies in nephrotic syndrome ${ }_{N}^{\omega}$ associated with extrarenal malignant disease. Lancet, $\sigma$ 2, 134-135.

Loughridge, L. W., and Lewis, M. G. (1971). Nephrotic syndrome in malignant disease of non-renal origin. 웅 Lancet, 1, 256-259.

Oldstone, M. B. A., Theofilopoulos, A. N., Gunvén,

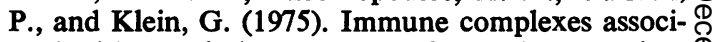
ated with neoplasia: presence of Epstein-Barr virus $\frac{\mathbb{}}{3}$ antigen-antibody complexes in Burkitt's lymphoma. O Intervirology, 4, 292-302.

Onyewotu, I. I., Holborow, E. J., and Johnson, G. D. $\vec{\bullet}$ (1974). Detection and radioassay of soluble circu- $\checkmark$ lating immune complexes using guinea-pig peritoneal exudate cells. Nature (Lond.), 248, 156-159. Poskitt, P. K. F., Poskitt, T. R., and Wallace, J. H. (1974). Renal deposition of soluble immune complexes in mice bearing B-16 melanoma. Journal of Experimental Medicine, 140, 410-425.

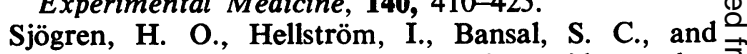
Hellström, K. E. (1971). Suggestive evidence that the 'blocking antibodies' of tumor-bearing indi- 3 viduals may be antigen-antibody complexes. Proceedings of the National Academy of Sciences, 68, 1372-1375.

Sjögren, H. O., Hellström, I., Bansal, S. C., Warner, 으 G. A., and Hellström, K. E. (1972). Elution of $\underset{\times}{\sim}$ 'blocking' factors from human tumors, capable of abrogating tumor-cell destruction by specifically 3 immune lymphocytes. International Journal of Cancer, 9, 274-283.

Steffelaar, J. W., De Graaff-Reitsma, C. B., and Feltkamp-Vroom, T. M. (1976). Immune complex $ᄋ$ detection by immunofluorescence on peripheral $\rightarrow$ blood polymorphonuclear leucocytes. Clinical and Experimental Immunology, 23, 272-278.

Stühlinger, W. D., Verroust, P. J., and Morel-Maroger, N L. (1976). Detection of circulating soluble immune $N$ complexes in patients with various renal diseases. N Immunology, 30, 43-47.

Theofilopoulos, A. N., Wilson, C. B., and Dixon, F. J. (1976). The Raji cell radioimmune assay for detect- $\bullet$ ing immune complexes in human sera. Journal of Clinical Investigation, 57, 169-182.

Requests for reprints to: Dr. H. M. Jansen, Department of Pulmonology, State University Hospital, Oostersingel 59, Groningen, The Netherlands. 